\title{
Fano resonances in antennas: General control over radiation patterns
}

\author{
Mikhail V. Rybin, ${ }^{1,2, *}$ Polina V. Kapitanova, ${ }^{2}$ Dmitry S. Filonov, ${ }^{2}$ Alexey P. Slobozhanyuk, ${ }^{2}$ Pavel A. Belov, ${ }^{2}$ \\ Yuri S. Kivshar, ${ }^{2,3}$ and Mikhail F. Limonov ${ }^{1,2}$ \\ ${ }^{1}$ Ioffe Physical-Technical Institute, St. Petersburg 194021, Russia \\ ${ }^{2}$ National Research University for Information Technology, Mechanics and Optics (ITMO), St. Petersburg 197101, Russia \\ ${ }^{3}$ Nonlinear Physics Center, Research School of Physics and Engineering, Australian National University, Canberra ACT 0200, Australia
}

(Received 6 August 2013; published 6 November 2013)

\begin{abstract}
The concepts of many optical devices are based on fundamental physical phenomena such as resonances. One of the commonly used devices is an electromagnetic antenna that converts localized energy into freely propagating radiation and vise versa, offering unique capabilities for controlling electromagnetic radiation. Here we propose a concept for controlling the intensity and directionality of electromagnetic wave scattering in radio-frequency and optical antennas based on the physics of Fano resonances. We develop an analytical theory of spatial Fano resonances in antennas that describes switching of the radiation pattern between the forward and backward directions, and we confirm our theory with both numerical calculations and microwave experiments. Our approach bridges the concepts of conventional radio antennas and photonic nanoantennas, and it provides a paradigm for the design of wireless optical devices with various functionalities and architectures.
\end{abstract}

DOI: 10.1103/PhysRevB.88.205106

PACS number(s): 78.20.Ci, 41.20.Jb, 84.40.Ba

\section{INTRODUCTION}

The communication flow transfused the modern world, and one of the key elements of current communication networks is an antenna that converts effectively the electromagnetic energy between localized and propagating waves. Since the first design of an antenna in 1886 by Heinrich Hertz, sizes, shapes, and materials of antennas have evolved dramatically, reflecting both human needs and technological progress. Along with the technological progress, a theory of antennas has been developed starting from the theory of radio-frequency antennas formulated around the middle of the 20th century.

The first decade of the 21st century demonstrates the need for global miniaturization, and nanotechnology makes it possible to scale down many electronic and photonic devices, thereby opening up new horizons for their applications. One such device is an optical nanoantenna that converts localized energy into freely propagating optical radiation and vise versa, offering unique capabilities for controlling electromagnetic radiation at the nanoscale. ${ }^{1}$ Nanoantennas are considered key elements in nanodevices being designed for a wide range of applications..$^{2-10}$ Besides, recent evidence suggests that a variety of organisms may harness an antenna for capturing sunlight and storing its energy transiently. ${ }^{11,12}$

Recent research activities in nanophotonics are mainly focused on the studies of plasmonic nanoantennas created by metal nanoparticles that increase the effectiveness of radiation or reception of signals of individual molecules or single quantum dots by several orders of magnitude, due to the Purcell effect. ${ }^{13-18}$ On the other hand, an optical nanoantenna should provide a low level of dissipation losses that is a serious weakness of all plasmonic devices. For this reason, several research teams turn their attention to the study of all-dielectric antennas with considerably low losses in the visible. ${ }^{19-21}$

The efficiency of any antenna can be enhanced substantially by resonance phenomena, which can shape and govern the structure of far-field radiation patterns. The resonances of different nature can be considered including the Bragg resonance associated with periodically arranged elements, i.e., based on a photonic crystal structure. ${ }^{22}$ However for problems addressed by single quantum objects, the most fruitful approach is to lower the antenna symmetry by adding some elements with resonance scattering. In the radio-frequency range, such antennas correspond to the concept proposed by Yagi based on a resonant element called $a$ reflector that forms a radiation pattern (the so-called Yagi-Uda antennas). ${ }^{23}$ To obtain a narrow radiation pattern, several elements referred to as directors are used additionally. ${ }^{23,24}$

Very different engineering solutions that define the antenna architecture can be reflected in the electromagnetic spectrum shown in Fig. 1. Here we refer to Yagi-Uda antennas that have been studied for the optical frequency range with metallic nanorods. ${ }^{2,6,25-30}$ Besides, the spherical metal particles and split-ring resonators have been suggested as elements of YagiUda antennas. ${ }^{31-34}$ We emphasize that all structure elements used so far in the antenna design are characterized by a resonant response to an external electromagnetic field which, as we show below, can be properly engineered.

Here we propose a concept for controlling the intensity and directionality of electromagnetic wave scattering for any antenna, including radio-frequency antennas and optical nanoantennas. Our concept is based on the physics of Fano resonance $^{35}$ that involves both constructive and destructive interference of a narrow frequency spectrum line with a broad spectral band. If two scattering channels have different locations in space, the relative phase shift depends on radiation directions that induce an extraordinary Fano resonance with the possibility of switching between the forward and backward radiation directions. The Fano resonance is observed when the wave scattering occurs through multiple channels; one of these channels possesses a narrow resonance in which the spectral dependence of phase undergoes a change by $\pi$, while other channels do not show a sharp change in the phase, forming the so-called continuum. Fano resonance has been observed across many different branches of physics, such as the studies of magnetization ${ }^{36}$ and electronic polarization phenomena, semiconductor and superconductor ${ }^{37,38}$ optics, as well as in 


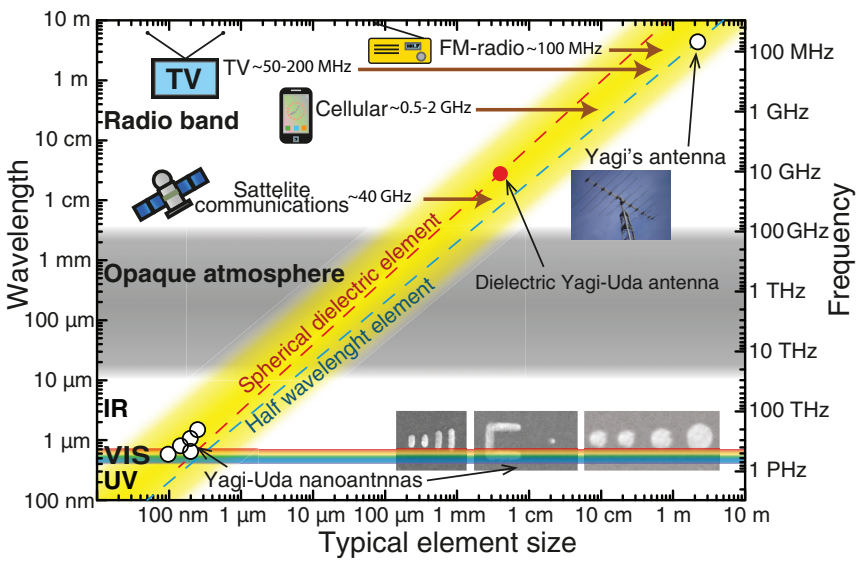

FIG. 1. (Color online) World of antennas. Examples of different devices with antennas and single antennas in the parameter plane antenna size vs operating frequency. Region of the typical size is marked by yellow. Circles show the results of different experiments with Yagi-Uda radio frequency and nanoantennas from Refs. 6,19, $23,25,27,28$, and 32 . The red dot marks our experimental data.

various nanoscale objects including photonic and plasmonic structures. ${ }^{39-45}$

We consider Fano resonance as an interference of two waves. The first one is proportional to $A e^{i \delta_{A}}(\Omega+i)^{-1}$ (the Lorenz function) and the second one is defined as $B e^{i \delta_{B}}$. Here $A, B$, and $\delta_{A, B}$ are real functions with relative changes in the frequency range of interest being negligible in comparison with the Lorenz function. Due to the interference, the resulting wave intensity can be written in the form

$$
I(\omega)=\left[\frac{(q+\Omega)^{2}}{1+\Omega^{2}} \eta+(1-\eta)\right] B^{2},
$$

where $q$ is the Fano asymmetry parameter, $\Omega=(\omega-$ $\left.\omega_{0}\right) /(\Gamma / 2)$ is the dimensionless frequency, $\omega_{0}$ is the central frequency, and $\Gamma$ is the width of the narrow band. An experimentally observed background component that does not interfere with the narrow band ${ }^{37,41,42}$ is accounted for by the introduction of an interaction coefficient $\eta \in[0 . .1]$ :

$$
\eta=\frac{2 F \cos ^{2} \delta}{F+2 \sin \delta+\sqrt{F^{2}+4 F \sin \delta+4}},
$$

where $F=A / B$ and $\delta=\delta_{A}-\delta_{B}$ are the relative intensity and phase difference, respectively. The Fano parameter of our special interest is

$$
q=\cos \delta F / \eta .
$$

Equation (1) shows that depending on the sign and value of $q$, the Fano-type spectra have four characteristic shapes. For special values of $q$ the narrow band is symmetrical ( $q=0$ and $q> \pm \infty$ ), while in the general case where $-\infty<q<0$ or $0<q<+\infty$, a narrow band exhibits an asymmetric profile, switching its shape into mirror image with changing of the sign of $q$.

Here we demonstrate that the unique flexibility of the narrow band leads naturally to a possibility for manipulating the spatial dispersion of the antennas' radiation. If resonant and nonresonant sources are placed asymmetrically relative to the antenna center, the Fano resonance will lead to the suppression of the radiation in some directions with the radiation enhancement in other directions. As a result, an asymmetric antenna with a resonance element will exhibit a dramatic modification of the radiation patterns and switching of the main lobe between the forward and backward directions. Here we consider a challenging case when the underlying mechanism of the Fano resonance leads to the transformation of the radiation pattern in the three-dimensional space while generally discussed Fano analysis is carried out for the spectral scale. $^{39,40,44,45}$ The Fano parameter $q$, which determines the shape of the narrow line, in the case of antennas becomes spatially dependent. We call the antenna whose operation is based on the physics of Fano resonances the Fano antenna.

\section{RESULTS}

\section{A. General approach based on the concept of Fano resonances}

First, we notice that for most types of antennas we can introduce a source of the radiation characterized by a dipole moment. Because of reciprocity, the source is usable for both emitting and absorbing radiation, and hereafter it is referred to as E/A feed. In an antenna, the electromagnetic wave undergoes a series of scattering events on the additional elements as well as on the E/A feed that leads to a change in the dipole moment of the E/A feed and in the response of the scatterers. A change of the dipole moment in the presence of a resonator is known as the Purcell effect, which depends on the geometry of the E/A feed. We do not consider a specific type of the E/A feed, and we normalize the dipole moment to unity. This normalization has no effect on the relative amplitude of the radiation propagating in different directions, i.e., on the far-field pattern.

Next, we introduce the second antenna element (director or reflector) having a resonant response (hereafter referred to as the $\mathrm{D} / \mathrm{R}$ element). As a result, the electromagnetic wave may reach the same far-field state via two different channels. The first traveling path from the E/A feed directly to the far-field zone corresponds to the formation of a broad background, where the wave phase and amplitude are nearly constant in the spectrum range of interest. The second traveling path includes the scattering event on a $\mathrm{D} / \mathrm{R}$ element having a resonant response and leads the formation of a narrow band, where the wave phase changes by $\pi$. If the amplitude of the narrow band can be approximated by the Lorenz function, the result of the interference of two waves in the far-field zone can be described by the Fano formula (1).

Departing from this general view and with the intention to further deepen our understanding of emitting and absorbing of electromagnetic waves in Fano antennas, a number of challenging problems can be formulated: How do we separate a Lorenz function for the amplitude of the narrow band for different real types of antennas? What determines the Fano asymmetry parameter $q$ ? And probably the most important question: Is it possible to switch the direction of the main lobe by changing the sign of the Fano asymmetry parameter $q$ at realistic geometric parameters of antennas?

In general, a real $\mathrm{D} / \mathrm{R}$ element can not be described by only one resonance, and there is a superposition of multipole moments. We will demonstrate that in most cases it is 
possible to separate an isolated dipole moment, which in the resonance region can be described by the Lorenz function multiplied by a slowly varying background originating from other multipole moments. Using an analytical model presented here, an optimal configuration of the Fano-antenna elements can be found in a first approximation, and parameters of the model can be fitted numerically using a nonlinear optimization method (e.g., Levenberg-Marquardt method). Note that the interaction between the E/A feed and the D/R element is taken into account by normalizing the E/A-feed dipole moment.

The most detailed theory has been developed for radio antennas. Traditional assumption about the description of radio antennas is based on the framework of effective electrical parameters, which depend on geometrical configuration of the antenna. ${ }^{24}$ The resonant frequency of the thin metal wire antenna corresponds to a first approximation to the half-wave antenna condition $L \approx \lambda / 2$, where $L$ is a length of the wire. For the long wire $L>\lambda / 2$, the impedance has mainly inductive character, and for the short wire $L<\lambda / 2$ the impedance has capacitive character. By contrast, here we consider the response of the antenna to the electric field, which in the case $L<\lambda$ can be described in terms of electric dipole momentum. ${ }^{46}$

To make the discussion more clear, we consider two regimes of the Fano-antenna operation depicted schematically in Fig. 2. A resonant $\mathrm{D} / \mathrm{R}$ element can have reflecting or directing properties depending on the phase of the response. Note that the condition for a wave propagation to the forward direction depends only on a phase shift $\Delta$ on the D/R element (Fig. 2). The requirements for the wave propagation to the backward direction are more complicated and depend both on the phase shift $\Delta$ and on the distance $d$ between the E/A feed and D/R element.

The D/R element fulfills the role of the director if (i) there is a forward constructive interference due to the phase shift $\Delta=0$ on the $\mathrm{D}$ element and (ii) a backward destructive interference due to the phase shift $\Delta=\pi$ on the $\mathrm{D}$ element and additional phase shift on the distance $d=\lambda / 4$. The D/R element fulfills the function of the reflector if (i) there is a forward destructive

(a)

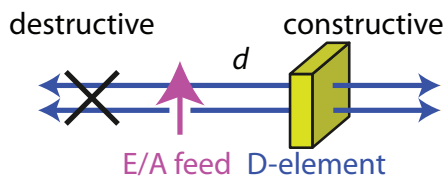

(b)

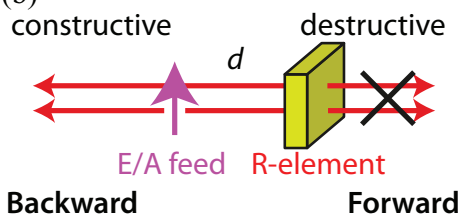

FIG. 2. (Color online) Schematic of the two regimes of Fano antennas. The antenna is composed of two elements: the emitting/absorbing element (E/A feed) and directing/reflecting element (D/R element). The upper diagram shows the directing interference regime and the lower diagram shows the reflecting interference regime. The distance between the E/A feed and D/R element is denoted by $d$. interference due to the phase shift $\Delta=\pi$ on the $\mathrm{R}$ element and (ii) a backward constructive interference due to the phase shift $\Delta=0$ on the $\mathrm{D}$ element and additional phase shift on the distance $d=\lambda / 4$. Unfortunately, the conditions for the optimal phase $(\Delta=0$ or $\Delta=\pi)$ are incompatible with the condition for the strong resonance response of a $\mathrm{D} / \mathrm{R}$ element that has the maximum at $\Delta=\pi / 2$. A tradeoff between the optimum conditions for a phase and an amplitude can be found by numerical methods.

There are too many antenna designs for us to describe them all here, and we must concentrate on the essentials. Many of the antenna designs share the same basic architecture and differ mainly in the size and number of D/R elements. We will discuss two of the most important D/R elements (a wire and a sphere) and conclude with an examination of different antennas types operating on the principles defined by the Fano resonance.

\section{B. Thin metal wire as a radio-frequency Fano antenna}

Let us consider the possibility of describing the far-field pattern of a radio antenna with a thin metal wire as a $D / R$ element by the Fano formula. It is particularly remarkable that in the seminal paper by Yagi $^{23}$ one can easily identify the Fano resonance profiles in a dependence of the current on the antenna's wire length $L$. Indeed, the emission pattern for an arbitrary set of dipoles can be represented in the form ${ }^{47,48}$

$$
c(\mathbf{n})=\sum_{i}\left(\left(\mathbf{I}-\mathbf{n} \mathbf{n}^{T}\right) \mathbf{P}_{i}-\sqrt{\frac{\mu_{0}}{\varepsilon_{0}}} \mathbf{n} \times \mathbf{M}_{i}\right) e^{-i k \mathbf{n} \mathbf{r}_{i}},
$$

where $\mathbf{P}_{i}$ and $\mathbf{M}_{i}$ are electrical and magnetic dipole moments of an antenna's elements with the position $\mathbf{r}_{i}$, where $k$ is the wave number, $\varepsilon_{0}$ and $\mu_{0}$ are the vacuum permittivity and permeability, respectively, and $\mathbf{n}$ is a unit vector in the direction of the radiation in the far field. It is possible to transform Eq. (4) into Fano equation (1) if we can separate a frequency range in which the spectral dependence of one dipole moment is well approximated by a resonant Lorenz function $A e^{i \delta_{A}}(\Omega-i)^{-1}$ and all others dipole moments are changing slowly and can be described by the function $B e^{i \delta_{B}}$. Importantly, the phase difference $\delta=\delta_{A}-\delta_{B}$ which determines the Fano parameter $q$ [Eq. (3)] possesses a strong directional dependence because all terms in Eq. (4) depend on the direction $\mathbf{n}$.

In the frequency range $L \approx \lambda / 2$, the thin wire antenna has an isolated electric dipole moment. ${ }^{46}$ The corresponding polarizability has a resonance feature, which can be expressed as a product of slowly varying background and the Lorenz function (see Appendix A). The resonance response is specified by a $\mathrm{Q}$ factor of $\omega_{0} / \Gamma \approx 10$. If there are only two dipoles (the E/A feed and D/R element), Eq. (4) can be simplified as

$$
c_{x z}(\varphi)=\cos \varphi+\left(a_{d} a^{e} \cos \varphi-c_{d} a^{m}\right) e^{-i k d \cos \varphi} .
$$

Here $\varphi$ is the angle between forward direction and the direction n, $a^{e}$ and $a^{m}$ are electrical and magnetic polarizability, $a_{d}=f_{d}\left(k^{2}-d^{-2}+i k d^{-1}\right), c_{d}=f_{d}\left(k^{2}+i k d^{-1}\right)$, and $f_{d}=$ $e^{i k d} / d$. For a thin wire antenna we have the relation $A e^{i \delta_{A}}(\Omega-$ $i)^{-1}=a_{d} a^{e} \cos \varphi e^{-i k d \cos \varphi}$ because the polarizability $a^{e}$ is proportional to the Lorenz function, $B=\cos \varphi$ and $\delta_{B}=0$. 

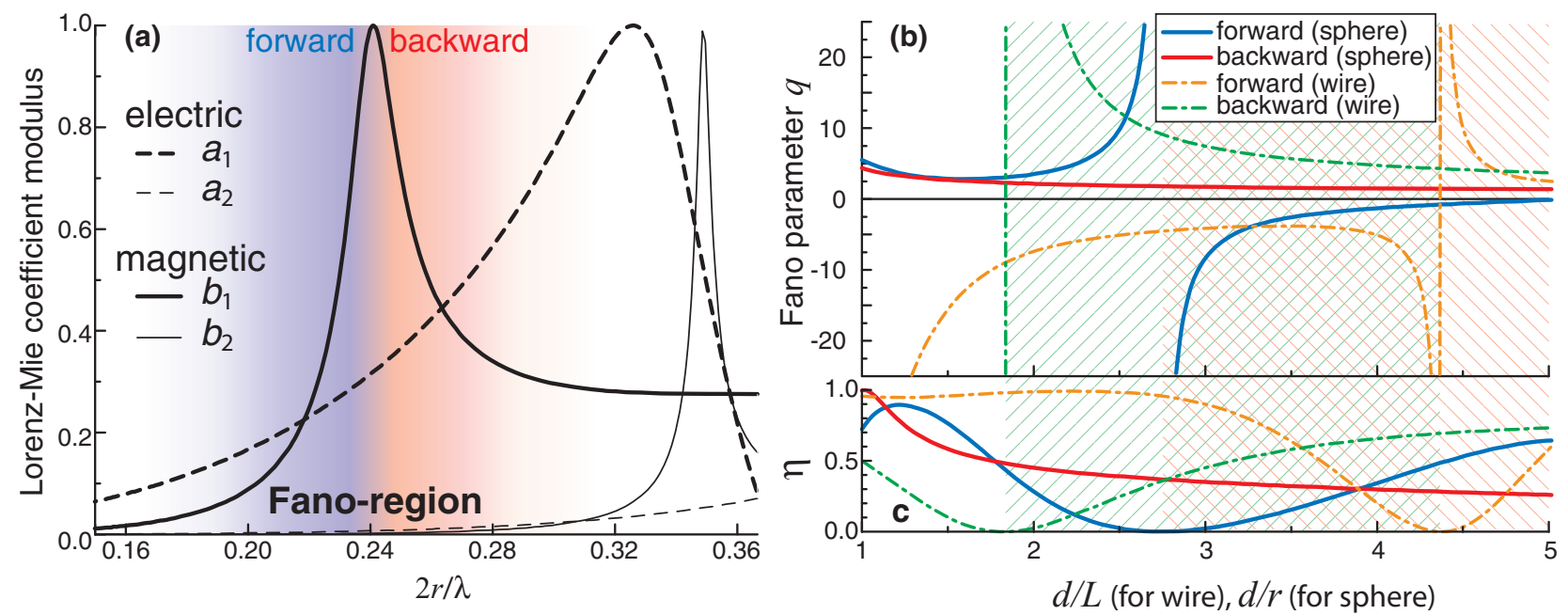

FIG. 3. (Color online) Fano regimes in antennas. (a) The Lorenz-Mie coefficient spectra of the lower multipole electric $\left(a_{1}, a_{2}\right)$ and magnetic $\left(b_{1}, b_{2}\right)$ moments of a single dielectric sphere with $\varepsilon=16$ versus the dimensionless frequency. In the spectral region of the Fano resonance between isolated magnetic dipole moment $b_{1}$ and slowly changing electric dipole moment $a_{1}$, blue indicates the region of the forward constructive interference and red indicates the region of the backward constructive interference in accordance with Fig. 2. (b), (c) The Fano parameter $q$ and the interaction coefficient $\eta$ as a function of the normalized distance $d / L$ (for a wire) or $d / r$ (for a sphere). Yellow and green shading suggest the intervals $2.75<d / r<5$ (for a sphere) and $1.8<d / L<4.3$ (for a wire) where the Fano parameter $q$ changes the sign for forward and backward emission.

In this case it is possible to yield the Fano parameter $q$ from Eq. (3).

As an illustration of the approach based on the Fano resonance, we are interested in an antenna configuration in which one frequency corresponds to the antenna radiating in the forward direction, and the other frequency corresponds to the antenna radiating in the backward direction (Fig. 2). Since the Maxwell equations are scalable with frequency and geometrical dimensions, we may reformulate the problem. Let us fix a configuration of the Fano antenna and find the operating frequency for the main lobe with forward and backward orientation. The reason is that the investigation of the spectral dependence is more instructive and convenient for the experimental realization. Moreover, in terms of the Fano resonance this problem has an obvious solution: An antenna configuration should provide a different sign of the Fano asymmetry parameter $q$ depending on the frequency of interest.

In the problem of a thin wire Fano antenna, the variable parameter is the distance $d$ between the E/A feed and D/R element (Fig. 2). The dependencies of the Fano parameter $q$ and the interaction coefficient $\eta$ on the normalized distance $d / L$ are presented in Fig. 3(b).

\section{Dielectric sphere as a Fano nanoantenna}

As a D/R element of the Fano antenna, we can consider not only a thin metallic wire, but also any other element having a resonant polarizability. Here we discuss a Fano antenna constructed from the all-dielectric spherical particles. ${ }^{19,21}$ The advantage of dielectric materials is the ability of a direct scaling down of the operating frequency to the visible range as the particle size is reduced. In contrast to the thin metallic wires, spherical particles have both electrical and magnetic moments within the range of the magnetic resonance for low frequencies [see Fig. 3(a)]. Therefore, unlike the case of metallic wires, in the Fano model for all-dielectric antennas the magnetic resonance plays the role of a narrow band. The corresponding $Q$ factor increases almost linearly with the increase of the dielectric constant. When the dielectric constant is about 10 , the $Q$ factor is close to 10 .

Here we will examine applicability of the Fano concept for describing the far-field pattern of all-dielectric antennas. We notice that the magnetic moment is pseudovector, and as a result the directivity pattern is formed even for zero spacing between the E/A feed and D/R element $(d=0)$. The magnetic moment is oriented in the $z$ direction. Therefore, in addition to the radiation pattern in the $x z$ plane, we consider the pattern in the $x y$ plane

$$
c_{x y}(\varphi)=1+\left(a_{r} a^{e}-c_{r} a^{m} \cos \varphi\right) e^{-i k d \cos \varphi} .
$$

With a large dielectric constant, the magnetic dipole resonance is standing out against the slowly varying electric resonance [see Fig. 3(a)]. Thus, in the $x y$ plane we have $A e^{i \delta_{A}}(\Omega-i)^{-1}=c_{r} a^{m} \cos \varphi e^{-i k d \cos \varphi}$ and $B e^{i \delta_{B}}=\cos \varphi+$ $a_{d} a^{e} \cos \varphi e^{-i k d \cos \varphi}$; more details are given in Appendix B. For the $x z$ plane, the similar equations have forms $A e^{i \delta_{A}}(\Omega-$ $i)^{-1}=c_{r} a^{m} \cos \varphi e^{-i k d \cos \varphi}$ and $B e^{i \delta_{B}}=1+a_{r} a^{e} e^{-i k d \cos \varphi}$. Now it is easy to obtain the Fano parameter $q$ and interaction coefficient $\eta$ from Eqs. (2) and (3). The corresponding dependencies as functions of the normalized distance $d / r$ are presented in Fig. 3(b).

Figure 4 summarizes our theoretical results. Intensity of the emission spectra calculated in the dipole approximation for selected high symmetry directions in the $3 \mathrm{D}$ space is presented in Fig. 4(a). The far-field radiation intensity in the $x z$ plane for the forward $(\varphi=0)$ and backward $\left(\varphi=180^{\circ}\right)$ directions is evaluated from Eq. (6). The condition when the Fano parameters for the forward and backward directions 

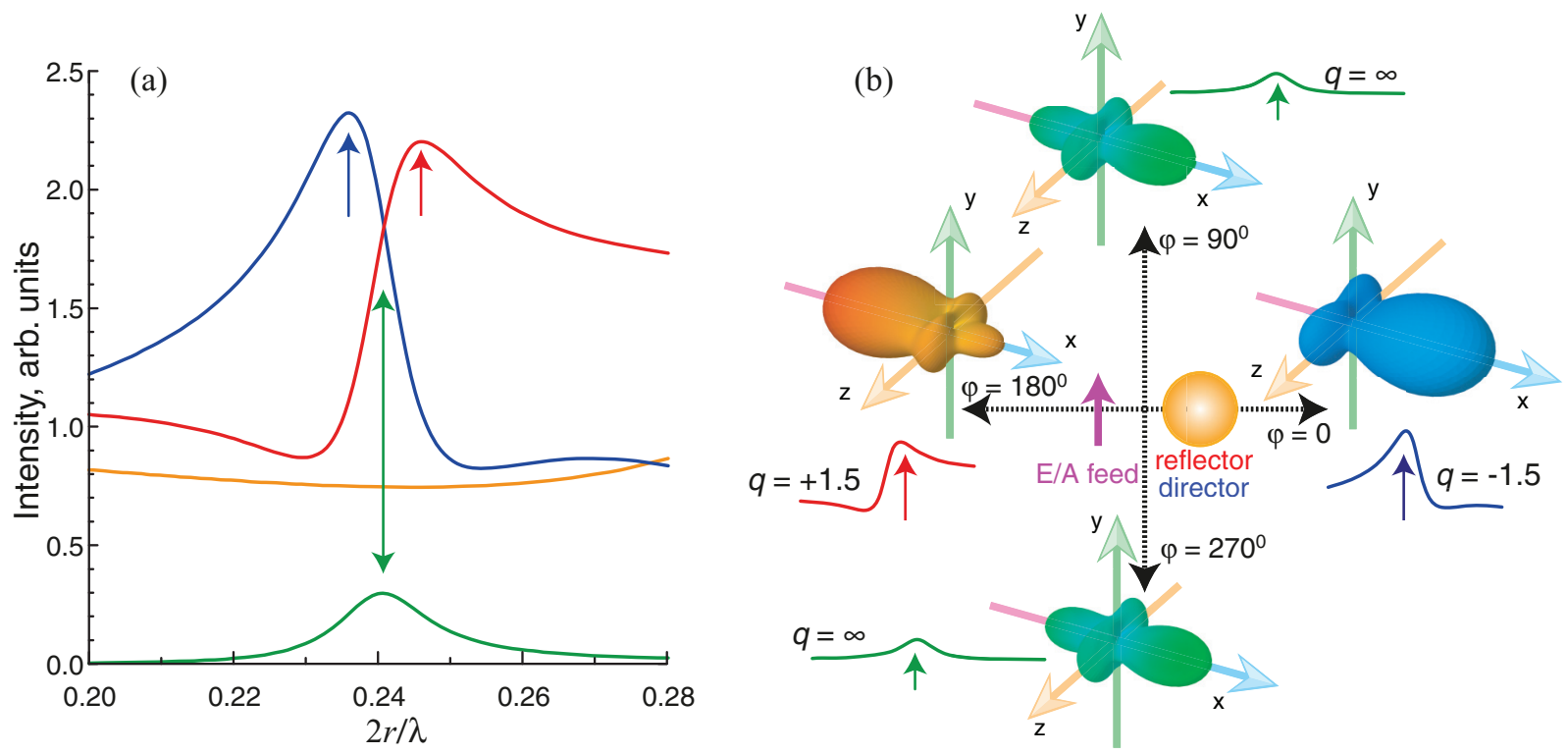

FIG. 4. (Color online) Spatial dependence of the Fano resonance and directivity patterns. (a) Spectral dependencies of the far-field radiation intensity along the $x$ axis for $\varphi=0$ (blue line) and $\varphi=180^{\circ}$ (red line), along the $y$ axis, for $\varphi=90^{\circ}$ and $270^{\circ}$ (green line) and along the $z$ axis (orange line) evaluated from Eqs. (5) and (6) for a single dielectric sphere with $\varepsilon=16$ and $d / r=3.5$. (b) The 3D far-field pattern for three selected frequencies marked in the panel (a) by arrows with corresponding colors. The Fano parameter $q$ for the main lobe is shown for each pattern.

have the same modules but different signs $(q= \pm 1.5)$ takes place at $d / r=3.5$ [Fig. 3(b)]. Figure 4 shows that at $d / r=$ 3.5 the maximum of the emission to the forward direction coincides with the minimum of the emission in the backward direction and vice versa. The 3D far-field pattern for three selected frequencies are marked in Fig. 4(a) by arrows with corresponding colors. The Fano parameter $q$ for the main lobe is shown for each pattern. The Fano-type switching of the main lobe is illustrated in Fig. 4(b) where the 3D far-field patterns are presented. The blue pattern (at $q=-1.5$ ) demonstrates the maximum emission in the forward direction at the frequency $2 r / \lambda=0.236$, whereas the red pattern (at $q=$ $+1.5)$ demonstrates the maximum emission in the backward direction at the frequency $2 r / \lambda=0.246$.

\section{Experimental observation of Fano resonance in all-dielectric antennas}

To estimate the performance of the Fano antenna, first we perform the full wave simulations of the antenna's response by employing the CST Microwave Studio. We consider the Fano antenna composed of a dielectric sphere and a source. We set the sphere permittivity and radius equal to $\varepsilon=16$ and $r=4 \mathrm{~mm}$, respectively. As the source, we model a short oscillator with the total length of $L=8 \mathrm{~mm}(l<\lambda / 4)$ and diameter of $a=2 \mathrm{~mm}$ excited by a point source in the middle. In numerical simulations, we adjust the distances between the sphere center and dipole to achieve an effective switching of the main lobe [see Figs. 5(a)-5(c)]. This switching occurs when the distance between sphere center and the dipole is $d=14 \mathrm{~mm}(d / r=3.5)$. The corresponding radiation patterns are depicted in the figure.

As the next step, we perform the experimental studies of the Fano antenna in an anechoic chamber. The dielectric sphere is made of $\mathrm{MgO}-\mathrm{TiO}_{2}$ ceramic characterized by the dielectric permittivity $\varepsilon=16$ and dielectric loss factor of $1.12 \times 10^{-4}$ measured in the frequency range $8-12 \mathrm{GHz}$. The sphere radius is $r=4 \mathrm{~mm}$ with the accuracy of $\pm 0.05 \mathrm{~mm}$. To feed the vibrator with the total length of $L=8 \mathrm{~mm}$, we employ a coaxial cable that is connected to an Agilent E8362C vector network analyzer. The Fano-antenna radiation patterns in the far field (at the distance $\cong 3 \mathrm{~m}, \cong 100 \lambda$ ) is measured by a horn antenna and rotating table. The effective switching of the Fano-antenna main lobe measured in the $x y$ and $x z$ planes has been found at the frequencies 9.06 and $9.55 \mathrm{GHz}$ [see Fig. 5(d)]. The measured value of the directivity is 3.5 at the frequencies 9.06 and $9.55 \mathrm{GHz}$. The measured characteristics agree very well with the numerically simulated results.

\section{DISCUSSION}

Our modern wireless society is based on different types of antennas having both traditional features and advanced functionalities including sensing, high-performance solar cells, energy communication between nanoemitters, quantum-dot pumping, etc. To meet a growing number of new demands, an antenna should possess versatile properties such as the ability of tuning the operating frequencies, receiving and transmitting electromagnetic waves in different directions. The concept of Fano antennas provides a unique opportunity to switch the direction of the main lobe and to communicate at different operating frequencies.

Now we can answer positively the question that was formulated above: using the results of our theoretical model we demonstrate experimentally that it becomes possible to switch the antenna's radiation direction by changing the sign of the Fano asymmetry parameter $q$ at realistic geometric parameters 

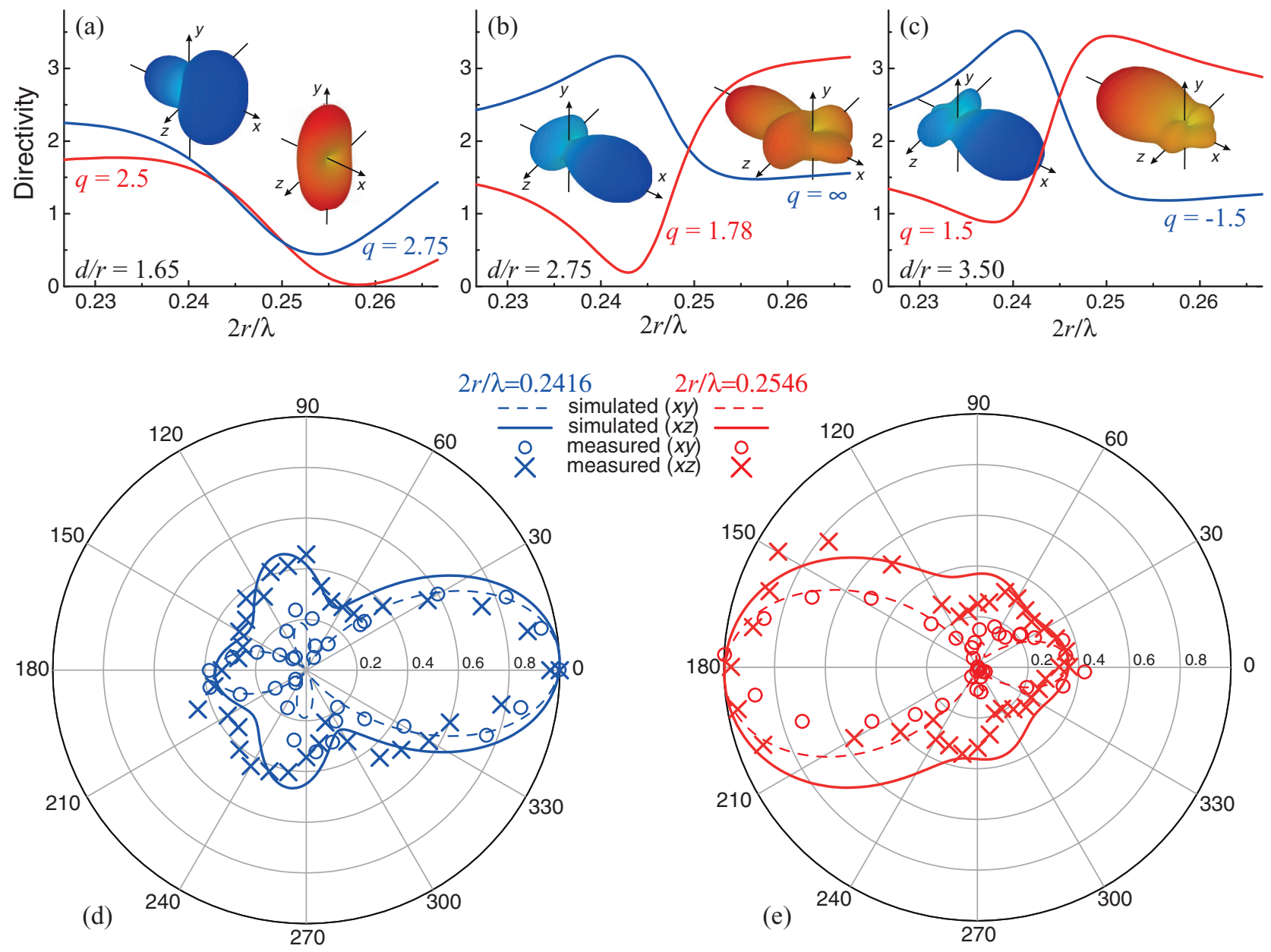

\section{$2 r / \lambda=0.2546$}

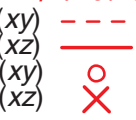

measured $(x y)$
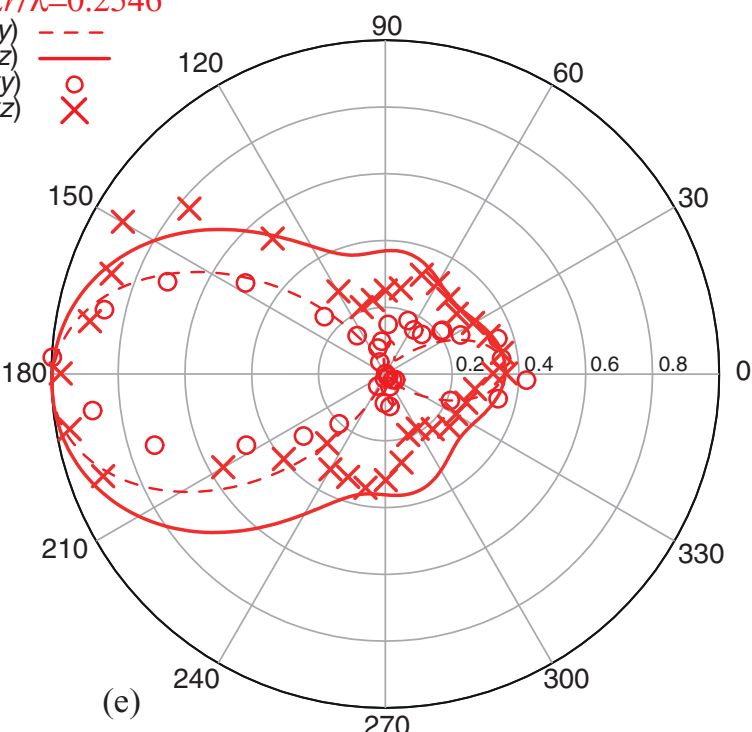

FIG. 5. (Color online) Experimental data and numerical calculations for the Fano effect in all-dielectric antenna. (a)-(c) Spectra of forward (blue lines) and backward (red lines) directivities of the all-dielectric antenna as a function of $d / r$ and the corresponding Fano parameter $q$ corresponding to Fig. 3(b). The 3D radiation patterns are presented for the frequencies $9.06 \mathrm{GHz}(2 r / \lambda=0.2416$, blue pattern) and $9.55 \mathrm{GHz}(2 r / \lambda=0.2546$, red pattern). The numerical results are obtained with the CST numerical simulations. (d),(e) Radiation patterns of the all-dielectric Fano antenna in the $x y$ and $x z$ planes at the frequencies $9.06 \mathrm{GHz}$ (d) and $9.55 \mathrm{GHz}$ (e) for $d / r=3.5$. The symbols indicate experimental data; solid and dashed lines show the results of numerical CST simulations.

of antennas. We present a simple analytical solution of the problem that can be used to design a tunable Fano antenna with required properties. To develop a Fano antenna, one needs to choose correctly the geometrical parameter $d / r$ for a sphere or the parameter $d / L$ for a wire. Specifically, Fig. 3(b) shows that the parameter $q$ changes its sign for the forward and backward emission within the intervals $2.75<d / r<5$ (for a sphere) and $1.8<d / L<4.3$ (for a wire). According to Eq. (1), for effective operation one needs a larger interaction coefficient $\eta$. Therefore, it is necessary to avoid the resonance regions of $q= \pm \infty$ at $d / r=2.75$ (for a sphere) and $d / L=1.8,4.3$ (for a wire), where $\eta$ vanishes [see Fig. 3(b)].

We emphasize the distinguished character of the Fano resonance discussed here. Usually the Fano resonance is considered for isotropic radiation or scattering and only in the spectral range without other variable parameters. ${ }^{35,39,40,44,45}$ In the case of Fano antennas with structural symmetry breaking, the parameter $q$ is related to the phase shifts in the forward and backward directions, and it has a pronounced directional dependence leading to an asymmetric far-field radiation pattern. For this reason, we introduce the variable parameter $\varphi$ that defines the spatial radiation direction. Consequently, the Fano parameter $q$ depends on $\varphi$ realizing the spatially dependent Fano resonance.

Finally, in a number of papers devoted to antennas, it is easy to recognize the characteristic Fano-like asymmetric profiles and the forward-backward switching effect discovered for different types of antennas, including Yagi-Uda radiofrequency antennas, ${ }^{23}$ all-dielectric antennas, ${ }^{19,21}$ nonlinear hybrid metal-dielectric nanoantennas, ${ }^{49}$ split-ring resonator optical antennas, ${ }^{33}$ and plasmonic nanoantennas. ${ }^{45,50-52} \mathrm{We}$ believe that our concept of Fano antennas employing the parameter $q$ can strongly expand the applications of Fano resonance in nanophotonics including different types of nanostructures such as oligomers ${ }^{44,45}$ and different functionalities such as sensing, optical communications, light generation, and routing at the nanoscale.

\section{ACKNOWLEDGMENTS}

The authors acknowledge useful discussions with A. E. Miroshnichenko. This work was supported by the Ministry 
of Education and Science of the Russian Federation (Project Nos. 11.G34.31.0020, 14.B37.21.0307, 14.B37.21.1964, and 01201259765) and the Government of St. Petersburg, as well as by research grants of Dynasty Foundation (Russia), Russian Foundation for Basic Research (Project Nos. 11-02-00865 and 13-02-00186), the Australian Research Council, as well as a Scholarship of President of Russian Federation for young scientists and graduate students.

\section{APPENDIX A: RADIO-FREQUENCY FANO ANTENNAS}

The electric polarizability of a thin-wire antenna is approximated by the Lorenz function multiplied by a slow varying function. We solve the inhomogeneous wave equation for the electric field by means of the dyadic Green function. ${ }^{53}$ Taking into account that the tangential component of the electric field vanishes at the antenna boundaries, we can express the scattering field in the form

$$
E_{y}^{\text {scat }}(\mathbf{r})=i \frac{\mu_{0} \omega}{k^{2}}\left(\frac{\partial^{2}}{\partial y^{2}}+k^{2}\right) \int d \mathbf{r}^{\prime} \frac{e^{i k\left|\mathbf{r}-\mathbf{r}^{\prime}\right|}}{4 \pi\left|\mathbf{r}-\mathbf{r}^{\prime}\right|} j_{y}\left(\mathbf{r}^{\prime}\right),
$$

assuming that the antenna is oriented along the $y$ axis. Here $E_{y}^{\text {scat }}(\mathbf{r})$ is the $y$ component of the scattered electric field, $\omega$ is the frequency, $c$ is the speed of light, $\mu_{0}$ is the magnetic constant, $k=\omega / c$ is the wave number, and $j_{y}$ is the current density. Using King's approximation, ${ }^{24}$ the integral is evaluated as a sum of real and imaginary functions, and the latter is responsible for the radiative decay. As a result, we find the current which allows us to estimate the electric polarizability of the antenna: ${ }^{46}$

$$
a^{e} \approx \frac{4 h}{\tilde{Z}_{0} k^{2} \pi} F(k h)
$$

where $F(x)$ is a function with the Lorenz function factor

$$
\begin{aligned}
F(x)= & \frac{\left(3 x+\sin x-3 i Z-8 \sin \frac{x}{2}\right)(1-\cos x)}{\left(3 i Z-\frac{3}{2} \pi-1\right)} \\
& \times \frac{1}{x-x_{0}+i \Gamma},
\end{aligned}
$$

and the parameters of the Lorenz function are

$$
\begin{gathered}
x_{0}=\frac{\pi}{2}-\frac{4\left(\frac{3}{2} \pi+1\right)}{9 Z^{2}+\left(\frac{3}{2} \pi+1\right)^{2}}, \\
\Gamma=\frac{12 Z}{9 Z^{2}+\left(\frac{3}{2} \pi+1\right)^{2}} .
\end{gathered}
$$

The impedance $\tilde{Z}$ reads

$$
\tilde{Z}_{0}=\ln \left[\left(\frac{\pi h}{2 a}\right)^{2}+1\right],
$$

where $a$ and $h$ are the wire radius and antenna's half-length, respectively.

\section{APPENDIX B: DIELECTRIC SPHERES}

It is known that for the frequencies not exceeding the frequency of the first electric dipole resonance, it is sufficient to take into account only the electric and magnetic dipole moments. ${ }^{54}$ The corresponding polarizability can be found from the Lorenz-Mie coefficients $a^{e}=3 i\left(2 k^{3}\right)^{-1} a_{1}$ and $a^{m}=$ $3 i\left(2 k^{3}\right)^{-1} b_{1}$, and they have the form

$$
\begin{aligned}
& a_{n}(x)=\frac{m \psi_{n}(m x) \psi_{n}^{\prime}(x)-\psi_{n}(x) \psi_{n}^{\prime}(m x)}{m \psi_{n}(m x) \zeta_{n}^{\prime}(x)-\zeta_{n}(x) \psi_{n}^{\prime}(m x)}, \\
& b_{n}(x)=\frac{\psi_{n}(m x) \psi_{n}^{\prime}(x)-m \psi_{n}(x) \psi_{n}^{\prime}(m x)}{\psi_{n}(m x) \zeta_{n}^{\prime}(x)-m \zeta_{n}(x) \psi_{n}^{\prime}(m x)},
\end{aligned}
$$

presented in terms of the Riccati-Bessel functions [real $\psi_{n}(x)$ and complex $\left.\zeta_{n}(x)\right]$. Here $m=n_{s} / n_{h}$ is the contrast of the refractive indices of the spherical particle $n_{s}$ and surrounding medium $n_{h}$, and the size parameter $x=k r$ is a product of the particle radius and the wave number.

The frequency of the first electric dipole resonance is about one and a half times higher than the frequency of the first magnetic dipole resonance. Thus, for sufficiently narrow resonances within the band of the magnetic dipole resonance, the electric polarizability does not change significantly [Fig. 3(a)]. Therefore, we can express the Lorenz-Mie coefficient $b_{1}$ as the product of the Lorenz function and slowly varying coefficient, namely

$$
b_{1}(x) \approx \frac{i \gamma e^{-i x}}{m}\left(\sin x \psi_{1}(m x)-m \psi_{1}(x) \sin m x\right) \frac{1}{1-i \Omega},
$$

where

$$
\begin{gathered}
\psi_{1}(x)=\frac{\sin x}{x}-\cos x, \\
\gamma=m-\frac{i}{\pi}\left(m^{2}-1\right), \\
\Omega=\left(x-x_{0}\right)|\gamma|^{2}, \\
x_{0}=\frac{\left(m^{2}-2\right)\left(m^{2}-1\right)+\pi^{2} m^{2}}{m \pi|\gamma|^{2}}, \\
\Gamma=\frac{2}{|\gamma|^{2}} .
\end{gathered}
$$

${ }^{4}$ M. Sivis, M. Duwe, B. Abel, and C. Ropers, Nat. Phys. 9, 304 (2013).

${ }^{5}$ E. S. Barnard, R. A. Pala, and M. L. Brongersma, Nat. Nanotech. 6, 588 (2011).

${ }^{6}$ P. Galliker, J. Schneider, H. Eghlidi, S. Kress, V. Sandoghdar, and D. Poulikakos, Nat. Commun. 3, 890 (2012). 
${ }^{7}$ M. W. Knight, H. Sobhani, P. Nordlander, and N. J. Halas, Science 332, 702 (2011).

${ }^{8}$ N. Large, M. Abb, J. Aizpurua, and O. L. Muskens, Nano Lett. 10, 1741 (2010).

${ }^{9}$ R. J. Moerland, T. H. Taminiau, L. Novotny, N. F. Van Hulst, and L. Kuipers, Nano Lett. 8, 606 (2008).

${ }^{10}$ A. A. Yanik, A. E. Cetin, M. Huang, A. Artar, S. H. Mousavi, A. Khanikaev, J. H. Connor, G. Shvets, and H. Altug, Proc. Natl. Acad. Sci. USA 108, 11784 (2011).

${ }^{11}$ N. Lambert, Y.-N. Chen, Y.-C. Cheng, C.-M. Li, G.-Y. Chen, and F. Nori, Nat. Phys. 9, 10 (2013).

${ }^{12}$ G. D. Scholes, Nat. Phys. 6, 402 (2010).

${ }^{13}$ M. S. Tame, K. R. McEnery, S. K. Ozdemir, J. Lee, S. A. Maier, and M. S. Kim, Nat. Phys. 9, 329 (2013).

${ }^{14}$ R. M. Bakker, H.-K. Yuan, Z. Liu, V. P. Drachev, A. V. Kildishev, V. M. Shalaev, R. H. Pedersen, S. Gresillon, and A. Boltasseva, Appl. Phys. Lett. 92, 043101 (2008).

${ }^{15}$ A. Kinkhabwala, Z. Yu, S. Fan, Y. Avlasevich, K. Müllen, and W. Moerner, Nat. Photon. 3, 654 (2009).

${ }^{16}$ S. Kühn, U. Håkanson, L. Rogobete, and V. Sandoghdar, Phys. Rev. Lett. 97, 017402 (2006).

${ }^{17}$ N. Liu, M. L. Tang, M. Hentschel, H. Giessen, and A. P. Alivisatos, Nat. Mater. 10, 631 (2011).

${ }^{18}$ T. H. Taminiau, R. J. Moerland, F. B. Segerink, L. Kuipers, and N. F. van Hulst, Nano Lett. 7, 28 (2007).

${ }^{19}$ D. S. Filonov, A. E. Krasnok, A. P. Slobozhanyuk, P. V. Kapitanova, E. A. Nenasheva, Y. S. Kivshar, and P. A. Belov, Appl. Phys. Lett. 100, 201113 (2012).

${ }^{20}$ B. Rolly, B. Stout, and N. Bonod, Opt. Express 20, 20376 (2012).

${ }^{21}$ Y. H. Fu, A. I. Kuznetsov, A. E. Miroshnichenko, Y. F. Yu, and B. Lukyanchuk, Nat. Commun. 4, 1527 (2013).

${ }^{22}$ Optical Properties of Photonic Structures: Interplay of Order and Disorder, edited by M. F. Limonov and R. M. De La Rue (CRC Press, Taylor \& Francis Group, 2012).

${ }^{23}$ H. Yagi, Proc. IRE 16, 715 (1928).

${ }^{24}$ R. W. P. King, G. J. Fikioris, and R. B. Mack, Cylindrical Antennas and Arrays, 2nd ed. (Cambridge Univ. Press, Cambridge, 2002).

${ }^{25}$ A. G. Curto, G. Volpe, T. H. Taminiau, M. P. Kreuzer, R. Quidant, and N. F. van Hulst, Science 329, 930 (2010).

${ }^{26}$ T. Kosako, Y. Kadoya, and H. F. Hofmann, Nat. Photon. 4, 312 (2010).

${ }^{27}$ J. Dorfmüller, D. Dregely, M. Esslinger, W. Khunsin, R. Vogelgesang, K. Kern, and H. Giessen, Nano Lett. 11, 2819 (2011).

${ }^{28}$ D. Dregely, R. Taubert, J. Dorfmüller, R. Vogelgesang, K. Kern, and H. Giessen, Nat. Commun. 2, 267 (2011).

${ }^{29}$ I. S. Maksymov, A. R. Davoyan, and Y. S. Kivshar, Appl. Phys. Lett. 99, 083304 (2011).

${ }^{30}$ I. S. Maksymov, A. E. Miroshnichenko, and Y. S. Kivshar, Opt. Express 20, 8929 (2012).
${ }^{31}$ J. Li, A. Salandrino, and N. Engheta, Phys. Rev. B 76, 245403 (2007).

${ }^{32}$ T. Coenen, E. J. R. Vesseur, A. Polman, and A. F. Koenderink, Nano Lett. 11, 3779 (2011).

${ }^{33}$ Y. Jie, Y. Li, and Y. Yonghong, Opt. Commun. 300, 274 (2013).

${ }^{34}$ J. Munárriz, A. V. Malyshev, V. A. Malyshev, and J. Knoester, Nano Lett. 13, 444 (2013).

${ }^{35}$ U. Fano, Phys. Rev. 124, 1866 (1961).

${ }^{36}$ V. Madhavan, W. Chen, T. Jamneala, M. F. Crommie, and N. S. Wingreen, Science 280, 567 (1998).

${ }^{37}$ M. F. Limonov, A. I. Rykov, S. Tajima, and A. Yamanaka, Phys. Rev. Lett. 80, 825 (1998).

${ }^{38}$ M. Limonov, S. Lee, S. Tajima, and A. Yamanaka, Phys. Rev. B 66, 054509 (2002)

${ }^{39}$ A. E. Miroshnichenko, S. Flach, and Y. S. Kivshar, Rev. Mod. Phys. 82, 2257 (2010).

${ }^{40}$ B. Luk'yanchuk, N. I. Zheludev, S. A. Maier, N. J. Halas, P. Nordlander, H. Giessen, and C. T. Chong, Nat. Mater. 9, 707 (2010).

${ }^{41}$ M. V. Rybin, A. B. Khanikaev, M. Inoue, K. B. Samusev, M. J. Steel, G. Yushin, and M. F. Limonov, Phys. Rev. Lett. 103, 023901 (2009).

${ }^{42}$ M. V. Rybin, A. B. Khanikaev, M. Inoue, A. K. Samusev, M. J. Steel, G. Yushin, and M. F. Limonov, Photon. Nanostruct.: Fundam. Applic. 8, 86 (2010).

${ }^{43}$ A. N. Poddubny, M. V. Rybin, M. F. Limonov, and Y. S. Kivshar, Nat. Commun. 3, 914 (2012).

${ }^{44}$ Y. Francescato, V. Giannini, and S. A. Maier, ACS Nano 6, 1830 (2012).

${ }^{45}$ M. Rahmani, B. Luk'yanchuk, and M. Hong, Laser. Photon. Rev. 7, 329 (2012).

${ }^{46}$ J. D. Jackson, Classical Electrodynamics, 3rd ed. (Wiley, New York, 1998).

${ }^{47}$ M. Born and E. Wolf, Principles of Optics, 7th ed. (Cambridge Univ. Press, Cambridge, 2006)

${ }^{48}$ A. B. Evlyukhin, C. Reinhardt, A. Seidel, B. S. Luk'yanchuk, and B. N. Chichkov, Phys. Rev. B 82, 045404 (2010).

${ }^{49}$ R. Noskov, A. Krasnok, and Y. S. Kivshar, New J. Phys. 14, 093005 (2012).

${ }^{50}$ R. Adato, A. A. Yanik, and H. Altug, Nano Lett. 11, 5219 (2011).

${ }^{51}$ N. Bonod, A. Devilez, B. Rolly, S. Bidault, and B. Stout, Phys. Rev. B 82, 115429 (2010).

${ }^{52}$ D. Vercruysse, Y. Sonnefraud, N. Verellen, F. B. Fuchs, G. Di Martino, L. Lagae, V. V. Moshchalkov, S. A. Maier, and P. Van Dorpe, Nano Lett. 13, 3843 (2013).

${ }^{53}$ R. G. Newton, Scattering Theory of Waves and Particles, International Series in Pure and Applied Physics (McGraw-Hill, New York, 1966).

${ }^{54}$ G. W. Mulholland, C. F. Bohren, and K. A. Fuller, Langmuir 10, 2533 (1994). 